\title{
Cytotoxicity of Benzyl Isoquinolinic Alkaloids, Palmatine and Corydaline on MCF-7 and MDA- MB231 Breast Cancer Cell Lines
}

DOI: $10.21859 /$ mci-supp-07

\author{
Younes Zarei ${ }^{1}$, Sakineh Kazemi Noureini ${ }^{1,2, *}$ \\ ${ }^{1}$ Department of Biology, Faculty of Basic Science, Hakim Sabzevari University, \\ Sabzevar, Iran \\ ${ }^{2}$ Medicinal Plants and Drugs Research Institute, Shahid Beheshti University, Tehran, \\ Iran \\ * Corresponding author: Sakineh Kazemi Noureini, Department of Biology, Faculty \\ of Basic Science, Hakim Sabzevari University, Sabzevar, and Medicinal Plants \\ and Drugs Research Institute, Shahid Beheshti University, Tehran, Iran. E-mail: \\ kazemibio@gmail.com
}

\section{Keywords:}

Breast Cancer

Palmatine

Corydaline

MCF-7

MDA-MB231 Cell Line

\begin{abstract}
Introduction: Breast cancer is the second and the most common leading cause of death in the world that has attracted special attention in drug design and discovery research projects. Here we investigated the cytotoxic effects of two closely related plant secondary metabolites from the family of benzyl isoquinolinic alkaloids on MCF-7 and MDA-MB231 cell lines.
\end{abstract}

Materials and Methods: Cytotoxicity of the derivatives in both MCF-7 and MDAMB231 cell lines were measured by using MTT test. Cells were seeded on 96 well plates and were treated with different concentrations of the alkaloid. After 48 hours, MTT was added at final concentration of $0.5 \mathrm{mg} / \mathrm{ml}$ and incubated for 4 hours. The purple dye developed by living cells was dissolved in DMSO and measured by Powerwave XS2 and Biotek plate reader. IC50 values were calculated by Gen5 software of the machine using at least 3 independent repeats of the experiment.

Results: MTT tests showed that with increasing concentration of these alkaloids, the percentage of cell survival significantly decreased. The obtained data indicated that Palmatine had an IC50 $=186 \mu \mathrm{M}$ in the MCF-7 cell line and $91 \mu \mathrm{M}$ in MDA-MB231 cell line. On the other hand, Corydaline with IC50 $=55 \mu \mathrm{M}$ in MCF-7 and $42 \mu \mathrm{M}$ in MDA-MB231 has a stronger cytotoxicity than Palmatine.

Conclusions: Comparing the MTT results, we find that corydaline was stronger than palmatine and it was slightly more cytotoxic in MDA-MB231 than MCF-7. 